\title{
Wavelength Scanning Interferometry for Topography of Microchannels at Roll-to-Roll Line with Optical Coherence Tomography
}

\author{
Janne Lauri \\ Optoelectronics and Measurement \\ Techniques Research Unit \\ University of Oulu \\ Oulu, Finland \\ janne.lauri@oulu.fi
}

\author{
Christina Liedert \\ VTT, Technical Research Centre of \\ Finland \\ Oulu, Finland \\ christina.liedert@screentec.com
}

\author{
Tapio Fabritius \\ Optoelectronics and Measurement \\ Techniques Research Unit \\ University of Oulu \\ Oulu, Finland \\ tapio.fabritius@oulu.fi
}

\begin{abstract}
Roll-to-roll (R2R) process is mass manufacturing method that can produce various products ranging from printed electronics to microfluidics. Microfluidics, used in biosensors, demands high tolerances and quality to guarantee correct functionality of sensors. In this work, optical coherence tomography device was installed at the R2R-line to measure the height and width of the hot embossed microchannel structures at speed of $1 \mathrm{~m} / \mathrm{min}$. The repeating channel structure on the $10 \mathrm{~m}$ long sample web was measured at $1 \mathrm{~m}$ interval. An algorithm, typically used in wavelength scanning interferometry (WSI), is utilized to recover topography at nanometer scale. The results showed that the R2R hot embossing process was very stable and predictable. If process parameters, for instance pressure at the hot embossing unit was increased, it resulted deeper channels as expected. The OCT, with the WSI algorithm, provides topography of microchannel at nanometer scale being thus an excellent tool for optimization of process parameters and for on-line quality control.
\end{abstract}

Keywords-wavelength scanning interferometry, nondestructive testing, online measurement, profilometer

\section{INTRODUCTION}

Roll-to-roll (R2R) process is an emerging method to high volume manufacture various components and systems ranging from printed electronics, solar cells, batteries to microfluidics. For instance, microfluidic components are typically used in biosensors requiring high detection repeatability and disposability. Thus, the high-volume manufacturing phase is very critical and should be monitored continuously to provide high yield and resource efficient production.

In $\mathrm{R} 2 \mathrm{R}$ process, microchannels are typically printed into a substrate by a process called hot embossing. In hot embossing, a typical web speed is $0.5-1.5 \mathrm{~m} / \mathrm{min}$ and feature sizes are in a range from few tens to hundreds of micrometers depending on the rheological characteristics of measured sample. Depths and widths of the features are depending on an embossing temperature and a pressure and the web speed. These parameters should be optimized especially at a beginning of a printing process as well as during, because of wear of a hot embossing mask. Thus, continuous, non-contact, high-speed topography monitoring at nanometers scale is required to assure high repeatability.

Commonly used high resolution topology measurement methods such as atomic force microscopy (AFM) or scanning electron microscope (SEM) are not feasible for

J.L is partially supported by European Regional Development Fund (project: PrintoCent10, no. A73286 and PrintoDiSe, no. A76026). T.F is partially supported by Academy of Finland's FIRI funding (grant no. 320017).

XXX-X-XXXX-XXXX-X/XX/\$XX.00 @20XX IEEE high-speed online measurements. That is why there is a need for developing alternative methods to bypass this problem.

For the non-contact surface profiling, there are numerous optical techniques, which are relatively fast and can achieve nanometers surface positioning resolution. Most of the techniques are interferometer based for instance phase shifting interferometry (PSI) [1], coherence scanning interferometry (CSI) [2, 3] and wavelength scanning interferometry (WSI) [4-7]. PSI and CSI typically require mechanical scanning and are therefore unsuitable for highspeed imaging applications [5]. WSI has an advantage over the CSI and PSI, instead of mechanical scanning the wavelength of the light source is scanned. This leads to higher imaging speeds. An alternative for the wavelength scanning and measuring the interference spectrum in time, is to use a broad band light source and measure the interference spectrum with a spectrometer. However, they are not capable to provide tomographic information about the sample.

In principle, WSI has lot of common with another 3D imaging technique, called optical coherence tomography (OCT) [8]. Beyond OCT's major application field in ophthalmology, it has emerged as a powerful tool in nondestructive testing of various types samples including microfluidics [9-11], displays [12], LEDs [13], printed electronics [14-17] and art work [18]. Development of high speed OCTs have led to two variants, the one is based on wavelength scanning (swept-source OCT) and the other is based on acquiring the whole spectrum at a single shot

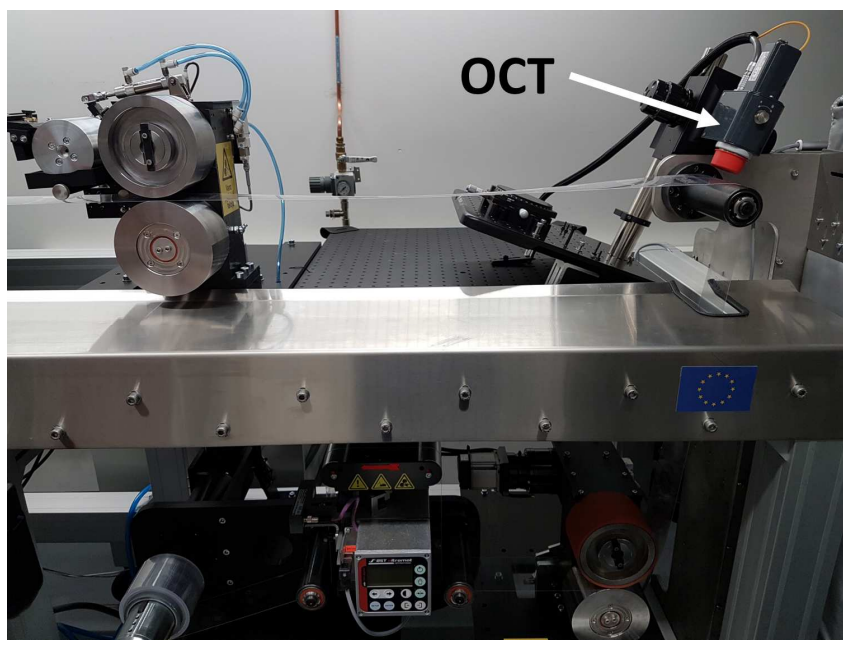

Fig. 1. OCT installed over the guiding roll in laboratory scale roll-to-roll printing and testing line. 
(spectral-domain OCT). Both, swept-source, and spectraldomain OCT, can achieve easily imaging frame rate over 100 frames per second. Due the similarities of these techniques, the methods applied in WSI for phase retrieval and an absolute optical path distance determination [19], have also been recently applied with OCT signal for topography of a cornea [20] and a solar cell inspection [21].

We have recently applied OCT for surface topography measurements at the R2R-line [22], however, in those measurement OCT suffered from the poor depth resolution due to the lack of WSI capability, and therefore, heights of the channels were not determined. In this study, we use a WSI algorithm to obtain surface topography at nanometerscale resolution without losing micrometer resolution tomography information. The widths and heights of channels are automatically determined from the obtained topographies. The stability of the R2R embossing process is shown and the applicability to detect changing process parameters is demonstrated.

\section{MATERIAL AND METHODS}

\section{A. $R 2 R$ unit and hot embossed sample}

The microchannels were hot embossed on thermoplastic polymethyl methacrylate (PMMA) film (Plexiglass 99524, Evonik Röhm, Germany), the width of $200 \mathrm{~mm}$ and thickness of $375 \mu \mathrm{m}$, at VTT (Technical Research Centre of Finland, Oulu, Finland) with the PIKO-pilot line. The length of the sample web was $10 \mathrm{~m}$. The embossing relief's width and height were $200 \mu \mathrm{m}$ and $80 \mu \mathrm{m}$ respectively. For the topography measurements of microchannels, the OCT device was installed online into another R2R-line (OPK-2017). Because of the different web width of the OPK-2017, the web was cut to the width of $8 \mathrm{~cm}$. To reduce the web vibrations, the measurements were performed over the guiding roll (see Fig. 1). Furthermore, to ensure a contact between the roller and the sample web, the OCT had to be tilted towards right. The web speed was set to $1 \mathrm{~m} / \mathrm{min}$, the same as the embossing speed.

\section{B. $O C T$}

A commercial OCT (Hyperion, Thorlabs Inc.) device was used to measure topography of the microfluidic structure at the R2R-line. The axial (depth) and the lateral resolution of the OCT were $5.8 \mu \mathrm{m}$ (in air) and $8 \mu \mathrm{m}$, respectively. The depth scanning range was $2.7 \mathrm{~mm}$. The maximum lateral scanning range of the system was $10 \mathrm{~mm}$ and was applied for cross direction (CD) scanning, i.e., across the web. From the $10 \mathrm{~m}$ long sample web, the same microfluidic pattern was measured at $1 \mathrm{~m}$ interval. Total of 11 measurement sets were acquired, each consisting of 500 cross-sectional images (Bscans). With the B-scan frame rate of $102 \mathrm{~Hz}$, it took approximately $5 \mathrm{~s}$ to capture 500 cross-sectional images. While acquiring the set of B-scans, the web moved $8.2 \mathrm{~cm}$ (perpendicular to the scanning direction). The B-scans consisted of 500 depth scans, so-called A-scans, and resulted a $20 \mu \mathrm{m}$ cross directional resolution. Along the web, in the machine direction (MD), the resolution of $163 \mu \mathrm{m}$ was defined from the web speed and the B-scan frame rate.

\section{Optical profilometer}

The reference measurements were performed with the optical profilometer (ContourGT-K, Bruker). The field of view with the $5 \mathrm{x}$ objective and 0.55 zoom lens was 2.302 $\mathrm{mm} \times 1.726 \mathrm{~mm}$. With the $640 \times 480$ pixels camera, the lateral pixel resolution was $3.596 \mu \mathrm{m}$. The device had automated stitching option to scan larger areas. The step height accuracy was $<0.75 \%$ for step heights $\geq 8 \mu \mathrm{m}$. The microchannel, measured with the OCT, was cut from the web, and measured offline by a profilometer on a damped optical table.

\section{Data processing}

To maximize imaging speed of the OCT, the raw camera data (interference spectra) was first buffered to a memory and then saved to hard drive after the measurement. Further data processing was performed offline in Matlab (R2019a). The script for raw data processing to obtain intensity data follows the same procedure as the original software. Shortly, the background intensity, obtained at beginning of every Bscan, was subtracted from the spectra. Then, spectrum was apodized with the Hanning window to improve the image quality. Before the Fourier transform (FFT) to z-space (spatial domain), the spectrum had to be resampled to be evenly spaced in wavenumber domain. This is because grating-based spectrometers acquire interference spectra as a function of a wavelength and not a wavenumber. After the FFT, a complex depth-resolved OCT signal was obtained together with the complex conjugate. WSI method was based on the finding the change in phase as a function of wavenumber. In OCT, an interference spectrum typically consists of multiple sinusoidal interference signals coming from different depths. To determine the phase slope from a
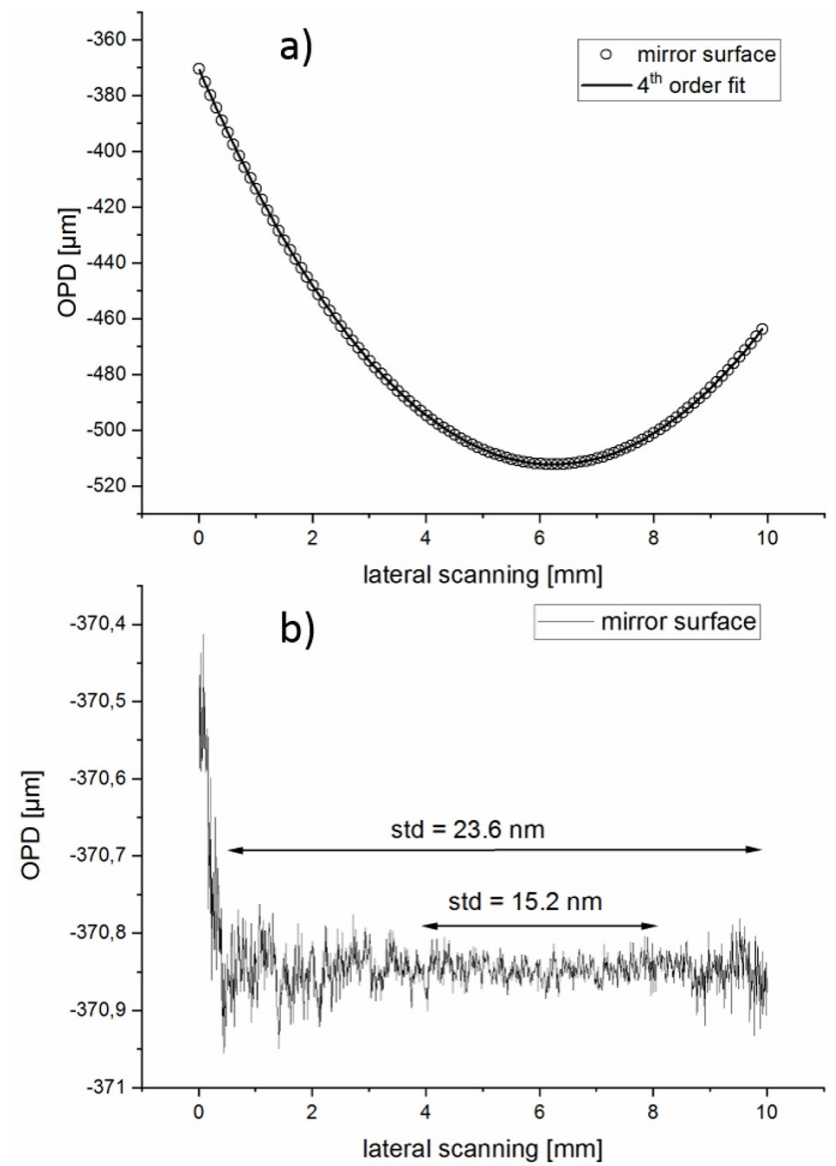

Fig. 2. Correction for a non-telecentric scanning: (a) the measured mirror surface with the $4^{\text {th }}$ order fitting (solid line) and (b) the mirror surface after correction. 
single frequency, corresponding a certain depth, in this case the surface, the surface must be segmented and filtered in Fourier domain. In this case the surface was found from the depth-resolved intensity signal based on the maximum intensity. The surface was band-pass filtered with the rectangular window. The filtered signal is then inverse Fourier transformed and the wrapped phase is obtained. Before defining the slope respect to the wavenumber $(\mathrm{k})$ the phase was unwrapped by adding multiples of $\pm 2 \pi$ when the phase difference between consecutive values was equal or greater than $\pi$. The slope of phase defines absolute optical path delay (OPD) respect to the reference mirror:

$$
O P D=\Delta \varphi / 2 \pi \Delta k,
$$

where $\varphi$ is the unwrapped phase. Due to the closed structure of the commercial device, the $\Delta \mathrm{k}$, needed for absolute OPD determination, was set by measuring $8 \mu \mathrm{m}$ high step height standard (VLSI Standards, Inc.). An advantage of the method compared to the phase-sensitive OCT, which suffers from $2 \pi$ ambiguity, is that OPD can be calculated for the whole depth range of the OCT at nanometers-scale resolution.

The utilized OCT suffers from a non-telecentric scanning, that causes geometrical field distortions to an image, e.g. flat surface appears to be convex or concave [23, 24]. The non-telecentric distortions (Fig. 2a) were corrected by a calibration curve, which was obtained from a flat mirror
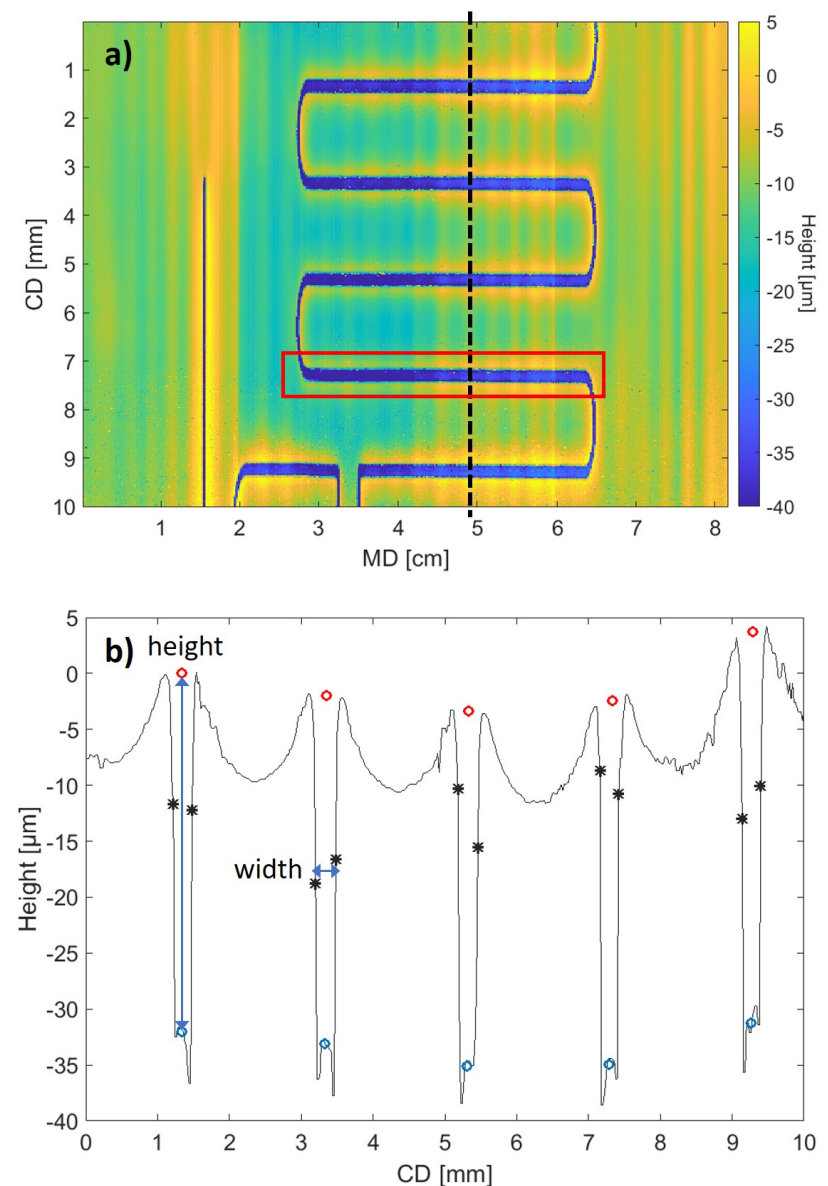

Fig. 3. Topography of the PMMA surface at the web speed of $1 \mathrm{~m} / \mathrm{min}$ (a). The rectangle points the channel which parameters are tracked along the web. The surface profile from the single frame along the dashed line with the locations for width (black crosses) and heigth (red and blue circles) determination (b). surface (PF10-03-P01, Thorlabs Inc, NJ, USA) with the surface flatness (peak to valley) of $\lambda / 10$ at $633 \mathrm{~nm}$. The number of measurement points were decimated for image clarity. The measured mirror surface was fitted with a $4^{\text {th }}$ order polynomial and used the correct the surface topography from the web (solid line in Fig. 2a). Figure $2 b$ shows the mirror surface after the non-telecentric scanning correction. There are some residual errors at the beginning of scan. These can be due the large scanning range, which can lead to optical aberrations at the far edge. In addition, the reduced signal-to-noise-ratio (SNR) at the edges, it will cause the increase of the standard deviation. At the center part of the scan (4 mm to $8 \mathrm{~mm}$ ), the standard deviation of the OPD was $15.2 \mathrm{~nm}$. From the scan range from $0.5 \mathrm{~mm}$ to $10 \mathrm{~mm}$ the standard deviation was $23.6 \mathrm{~nm}$. Thus, the surface positioning sensitivity, quantified by the standard deviation of the OPD at good SNR conditions, was in the nanometersrange.

After the distortion correction, the microchannel's surface was a median filtered to remove outliers and peaks, especially at channels edges, where signal is poor or completely lost. In addition, a possible residual tilt was corrected by the line fitted to the surface.

Rapid changes in the surface position at the channel's walls were used for locating channels and walls. The number and the preliminary locations of walls of channels in the Bscan were defined by finding the largest differences in the neighboring depth pixel followed by Matlab peak detection routine ('findpeaks'). Each found peak corresponded an estimated wall location. The wall locations were used the find the top and bottom parts of the channels. The top was defined as a mean from the maximum heights on left and right side of the microchannel. The bottom was an average from the bottom part. The determined microchannel's height was the difference between the top and bottom. For the microchannel's width determination, the position of the wall pixels was optimized to be at full width at half maximum or as close as possible in the limits of the lateral pixel resolution of $20 \mu \mathrm{m}$.

\section{RESULTS}

Figure 3a shows the topography of the scanned PMMA surface at the web speed of $1 \mathrm{~m} / \mathrm{min}$. During the time it took to acquire 500 frames in $\mathrm{CD}$ direction, the web moved 8.2 $\mathrm{cm}$. The vertical lines are caused by the vibrations of the $\mathrm{R} 2 \mathrm{R}$ machine and the moving web. However, the frequency of the vibrations was low and did not influence the surface topography at the fast-scanning direction (CD). The frame rate, and resulting MD resolution of $163 \mu \mathrm{m}$, was too low to resolve profile of the microchannels at the bends. Higher frame rate and better MD resolution can be obtained by decreasing the number of A-scans in the frame, decreasing the web speed or utilize a faster OCT device. Figure $3 \mathrm{~b}$ shows the profile from the single frame obtained at the black dashed line in Fig. 3a. The red and blue circles show the determined top and bottom of the microchannel, respectively. The height is the difference between the top and bottom. The width is determined, in the limits of the lateral measurement points, from the black crosses. The elevated heights next to microchannel's wall are typical for R2R embossing, in which time for embossing is very short. This results that the PMMA does not have enough time to take the shape of the master mold. 


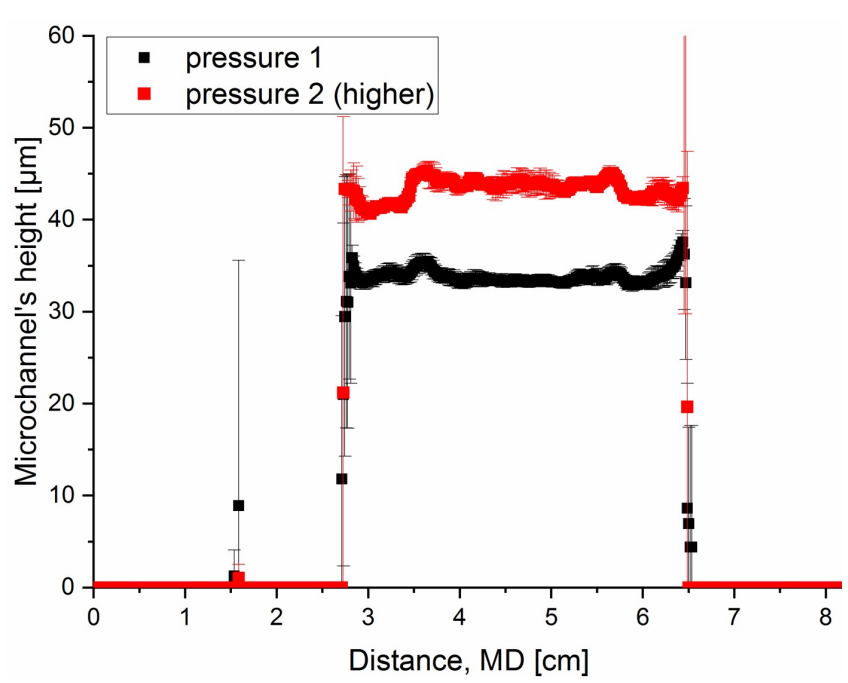

Fig. 4. Average microchannel's height with the standard deviation determined from the 9 measurements at $1 \mathrm{~m}$ interval (black rectangles, pressure 1), and after increasing the hot embossing pressure from two measurements at $1 \mathrm{~m}$ apart (red rectangles, pressure 2).

While the web was moving its position was continuously adjusted in the $\mathrm{CD}$ direction by the roll-to-roll machine. Because OCT's measurement location was constant, the position of the tracked microchannel pattern in the $C D$ direction changed between acquisitions. This resulted that the number of microchannels in the topography images varied from 2-5. Therefore, only one microchannel, depicted by a red rectangle in Fig. 3b, was chosen for further analyses. In addition to shift in $\mathrm{CD}$ direction, the location of the microchannel in MD direction varied due to the manual triggering. Before the comparison, 9 channels (measured at 1 $\mathrm{m}$ interval) were aligned with each other by calculating the shift with a cross-correlation function. Fig. 4 shows the average microchannel's height from the 9 measurements with the standard deviation (black rectangles, pressure 1). The average height (from 3 to $6 \mathrm{~mm}$ ) was $33.7 \pm 0.8 \mu \mathrm{m}$. After this the embossing pressure was increased and printing was continued. Unfortunately, in this sample web only $2 \mathrm{~m}$ of microchannels were hot embossed at higher pressure, thus, only two topography measurements were acquired at the interval of $1 \mathrm{~m}$. The red rectangles show the height of the microchannel at the higher pressure (pressure 2). As expected, the microchannel's height was higher with the increased embossing pressure. The average height (from 3 to $6 \mathrm{~mm}$ ) was $43.5 \pm 0.7 \mu \mathrm{m}$. Interestingly, there was a repeating variation pattern in the heights between the microchannels, for instance the rise at the $3.6 \mathrm{~cm}$. This was most likely originated already from the embossing mask, but the mask profile was not available for further measurements to confirm this observation.

In addition to the height, also the widths were determined at both hot embossing pressures. Fig. 5 shows the average widths at pressure 1 (black line) and pressure 2 (red line). The increased pressure did not result an increased width in the limits of the measurement resolution. The position of the microchannel was different compared to the Fig. 4 because it was aligned to the other channel.

After the R2R measurements, the single pattern was cut out from the web and measured with the optical profilometer on top of the damped optical table. Due to limited field of view $(2.3 \times 1.7 \mathrm{~mm})$ of the profilometer, multiple

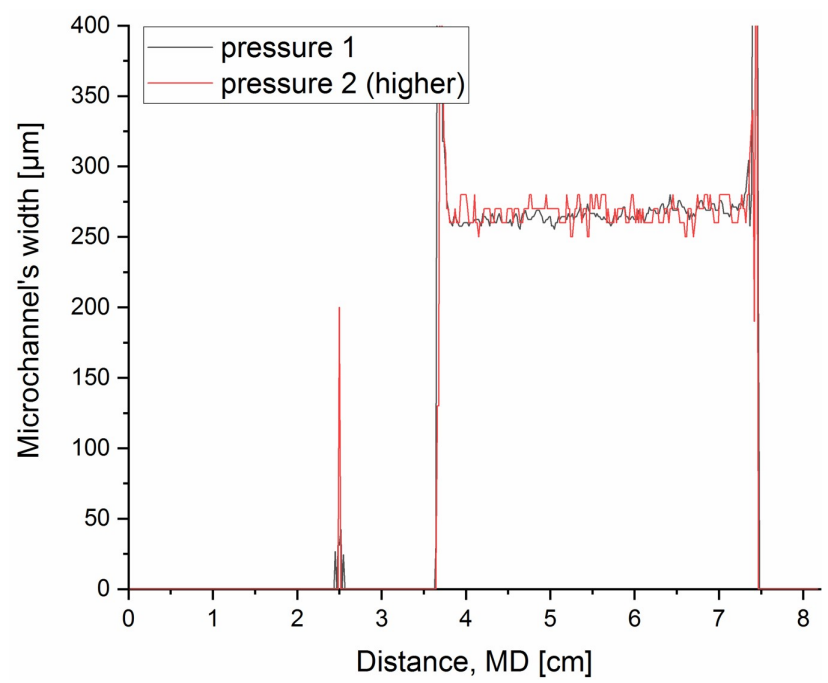

Fig. 5. Average microchannels' widths at two different pressures.

measurements and stitching of images had to be applied. The total imaged area was 13 × $5 \mathrm{~mm}$. Figs. $6 \mathrm{a}$ and $6 \mathrm{~b}$ show the surface profiles measured with the OCT and optical profilometer, respectively. The tilts were removed, and the absolute values were subtracted by the mean. The optical profilometer suffers also for the lost signal at the microchannel's edges. The correspondence with the reference topography was good and the heights of the microchannels are comparable. The reference profile had less noise, which could be due to the reason that measurements were performed on the damped optical table that reduced
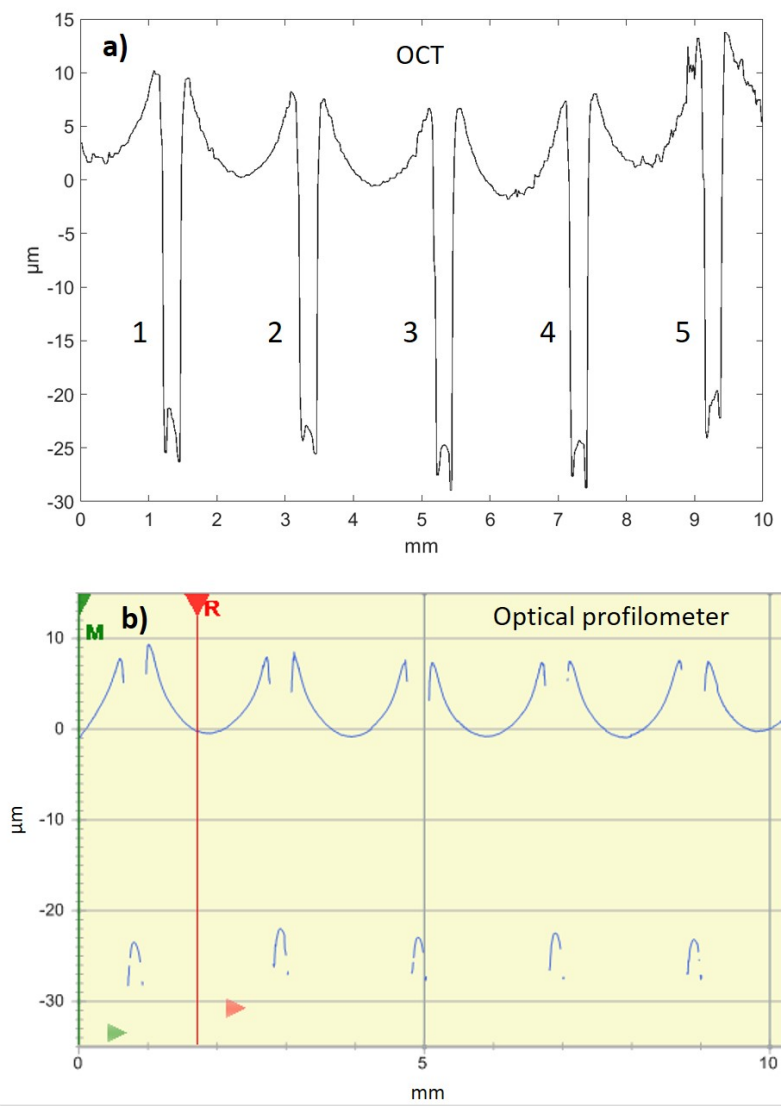

Fig. 6. Channel surface profiles measured with the OCT (a) and the optical profilometer ContourGT-K (b). The dimensions of the channels are shown in Table 1. 
TABLE I.

DETERMINED HEIGTS AND WIDTHS ALONG 5 MM DISTANCE FROM THE SAME SAMPLE. OCT $(\mathrm{N}=31)$ AND CONTOURGT-K $(\mathrm{N}=1390)$. SEE FIG. 6. FOR CHANNEL NUMBERING.

\begin{tabular}{|c|c|c|c|c|}
\hline \multirow{2}{*}{ Channel } & \multicolumn{2}{|c|}{ OCT } & \multicolumn{2}{|c|}{ ContourGT-K } \\
\hline & Height [ $\mu \mathrm{m}]$ & Width [ $\mu \mathrm{m}]$ & Height [ $\mu \mathrm{m}]$ & Width [um] \\
\hline 1 & $32.09 \pm 0.36$ & $277.5 \pm 8.4$ & $32.45 \pm 0.46$ & $283.1 \pm 4.9$ \\
\hline 2 & $31.64 \pm 0.42$ & $275.6 \pm 12.2$ & $32.16 \pm 0.50$ & $285.0 \pm 5.3$ \\
\hline 3 & $31.76 \pm 0.33$ & $269.4 \pm 12.4$ & $31.92 \pm 0.51$ & $279.1 \pm 4.6$ \\
\hline 4 & $32.44 \pm 0.43$ & $261.3 \pm 4.9$ & $32.54 \pm 0.58$ & $283.3 \pm 5.2$ \\
\hline 5 & $34.45 \pm 0.74$ & $266.9 \pm 14.9$ & $33.39 \pm 0.87$ & $276.9 \pm 5.3$ \\
\hline
\end{tabular}

vibrations compared to the online measurements at the $\mathrm{R} 2 \mathrm{R}$ line. Table I shows the microchannels' averaged heights and widths with the standard deviations calculated from the 5 $\mathrm{mm}$ distance at the same location of the pattern. The channel numbers correspond the numbering in Fig 6a. The number of the measured lines along $5 \mathrm{~mm}$ distance was 31 for the OCT and 1390 for the optical profilometer. This was due the difference in MD resolution $(163 \mu \mathrm{m}$ vs. $3.596 \mu \mathrm{m})$. The average height is slightly smaller in the OCT measurement expect for the channel number 5 . The difference could result from the calibration of the devices, although the same step height standard was used for the calibration of both devices. Other factors that can affect are the vibrations of the web, different tilting of the sample and the SNR. The standard deviations are comparable, even though, the OCT measurements were performed from the vibrating and moving web. Because of the poorer $\mathrm{CD}$ resolution of the OCT $(20 \mu \mathrm{m}$ vs. $3.596 \mu \mathrm{m})$, the width values deviate more, and standard deviations are higher for OCT.

\section{CONCLUSION}

OCT was successfully applied to define surface characteristics of the moving microchannel at the R2R-line. The WSI processing algorithm was applied to determine surface position at nanometer-scale resolution. This improved the resolution compared to the method based on only the maximum intensity [22], of which resolution was, without zero padding, $2.7 \mu \mathrm{m}(2.7 \mathrm{~mm} / 1024)$. The heights and widths were automatically determined for two different hot embossing pressures. The stability of the hot embossing process was good, and when the embossing pressure was increased it was immediately seen as the increased height of the microchannel. The higher pressure did not influence on the width of the microchannel. The vibration of the R2Rmachine and the moving web did not influence the width and height determination. The vibrations were only seen at the MD direction.

The reference measurements, obtained with the commercial optical profilometer, showed excellent agreement with the OCT results. The noise in reference measurements was smaller, however, it was most likely due to the reason that they were measured on the vibration damped optical table.

The bends of the microchannel pattern could not be resolved due to the limited frame rate of the OCT, however, there are much faster OCT systems currently availably to solve the issue. In this study, the data processing time was not optimized for a real time online process monitoring. For the optimization, the utilization of graphical processing units would provide enough speed for online applications.
We demonstrated that OCT with the WSI algorithm provides high-speed, nanometer-scale resolution tool for online topography and quality characterization of the hot embossed microchannel structures in R2R-line. As 3D imaging modality, OCT would also resolve microchannel's real height after lidding process with nanometers resolution. This would not be feasible for the techniques meant exclusively for surface topography measurements.

\section{REFERENCES}

[1] P. de Groot, "Principles of interference microscopy for the measurement of surface topography," Adv. Opt. Photon., vol. 7, pp. 1$65,2015$.

[2] L. Deck and P. de Groot, "High-speed noncontact profiler based on scanning white-light interferometry," Appl. Optics, vol. 33, pp. 73347338, 1994

[3] B.S. Lee and T.C. Strand, "Profilometry with a coherence scanning microscope," Appl. Opt., vol. 29, pp. 3784-3788, 1990.

[4] I. Yamaguchi, A. Yamamoto and M. Yano, "Surface topography by wavelength scanning interferometry," Opt. Eng., vol. 39, pp. 40-46, 2000 .

[5] G. Moschetti, A. Forbes, R.K. Leach, X. Jiang and D. O’Connor, "Phase and fringe order determination in wavelength scanning interferometry," Opt. Express, vol. 24, pp. 8997-9012, 2016.

[6] V. Lédl, P. Psota, F. Kaván, O. Matoušek and P. Mokrý, "Surface topography measurement by frequency sweeping digital holography," Appl. Optics., vol. 56, pp. 7808-7814, 2017.

[7] D. Xiaoli and S. Katuo, "High-accuracy absolute distance measurement by means of wavelength scanning heterodyne interferometry," Meas. Sci. Technol., vol. 9, pp. 1031-1035, 1998.

[8] A.F. Fercher, W. Drexler, C.K. Hitzenberger and T. Lasser, "Optical coherence tomography - principles and applications," Rep. Prog. Phys., vol. 66, pp. 239-303, 2003.

[9] J. Lauri, J. Czajkowski, R. Myllylä and T. Fabritius, "Measuring flow dynamics in a microfluidic chip using optical coherence tomography with $1 \mu \mathrm{m}$ axial resolution," Flow Meas. Instrum., vol. 43, pp. 1-5, 2015.

[10] L. Wang, W. Xu, M. Bachman, G.P. Li and Z. Chen, "Phase-resolved optical Doppler tomography for imaging flow dynamics in microfluidic channels," Appl. Phys. Lett., vol. 85, pp. 1855-1857, 2004.

[11] J. Lauri, C. Liedert, A. Kokkonen and T. Fabritius, "Effect of solvent lamination on roll-to-roll hot-embossed PMMA microchannels evaluated by optical coherence tomography," Mater. Res. Express, vol. 6, pp. 075333, 2019.

[12] S. Kim, J. Kim and S. Kang, "Nondestructive defect inspection for LCDs using optical coherence tomography," Displays, vol. 32, pp. 325-329, 2011.

[13] N.H. Cho, U. Jung, S. Kim and J. Kim, "Non-destructive inspection methods for LEDs using real-time displaying optical coherence tomography," Sensors, vol. 12, 2012.

[14] E. Alarousu, A. AlSaggaf and G.E. Jabbour, "Online monitoring of printed electronics by spectral-domain optical coherence tomography," Sci. Rep., vol. 3, pp. 1562, 2013.

[15] J. Czajkowski, J. Lauri, R. Sliz, P. Falt, T. Fabritius, R. Myllyla and B. Cense, "Sub-micron resolution high-speed spectral domain optical coherence tomography in quality inspection for printed electronics," Proc. SPIE 8430, Optical Micro- and Nanometrology IV, pp. 84300K-8, 2012.

[16] J. Czajkowski, P. Vilmi, J. Lauri, R. Sliz, T. Fabritius and R. Myllyla, "Characterization of ink-jet printed RGB color filters with spectral domain optical coherence tomography," Proc. SPIE 8493, Interferometry XVI: Techniques and Analysis, pp. 849308-7, 2012

[17] J. Czajkowski, T. Fabritius, J. Ulański, T. Marszałek, M. GazickiLipman, A. Nosal, R. Śliż, E. Alarousu, T. Prykäri, R. Myllylä and G. Jabbour, "Ultra-high resolution optical coherence tomography for encapsulation quality inspection," Appl. Phys. B, vol. 105, pp. 649657, 2011.

[18] H. Liang, M.G. Cid, R.G. Cucu, G.M. Dobre, A.G. Podoleanu, J. Pedro and D. Saunders, "En-face optical coherence tomography - a novel application of non-invasive imaging to art conservation," Opt. Express, vol. 13, pp. 6133-6144, 2005.

[19] M. Takeda, H. Ina and S. Kobayashi, "Fourier-transform method of fringe-pattern analysis for computer-based topography and interferometry," J. Opt. Soc. Am., vol. 72, pp. 156-160, 1982. 
[20] S. Das, C. Liu, M. Singh, M.D. Twa and K.V. Larin, "Modified wavelength scanning interferometry for simultaneous tomography and topography of the cornea with Fourier domain optical coherence tomography," Biomed. Opt. Express, vol. 9, pp. 4443-4458, 2018.

[21] M. Tsai, F. Chang, Y. Yao, J. Mei and Y. Lee, "Optical inspection of solar cells using phase-sensitive optical coherence tomography," Sol. Energ. Mat. Sol C, vol. 136, pp. 193-199, 2015.

[22] J. Lauri, C. Liedert, R. Liedert and T. Fabritius, "Online quality assurance of microchannels in roll-to-roll by optical coherence tomography," in - 2020 IEEE International Instrumentation and Measurement Technology Conference (I2MTC), pp. 1-5, 2020.

[23] V. Westphal, A.M. Rollins, S. Radhakrishnan and J.A. Izatt, "Correction of geometric and refractive image distortions in optical coherence tomography applying Fermat's principle," Opt. Express, vol. 10, pp. 397-404, 2002.

[24] M. Ourak, B. Tamadazte, G. J. Laurent and N. Andreff, "Geometric calibration of an OCT imaging system," 2018 IEEE International Conference on Robotics and Automation (ICRA), pp. 3993-3999, 2018 . 